\title{
A!
}

This is an electronic reprint of the original article.

This reprint may differ from the original in pagination and typographic detail.

Islam, SK Firoz; Zyuzin, Alexander

\section{Collective modes in an imbalanced nodal-line semimetal}

Published in:

Physical Review B (Condensed Matter and Materials Physics)

DOI:

10.1103/PhysRevB.104.245301

Published: 15/12/2021

Document Version

Publisher's PDF, also known as Version of record

Please cite the original version:

Islam, SK. F., \& Zyuzin, A. (2021). Collective modes in an imbalanced nodal-line semimetal. Physical Review B (Condensed Matter and Materials Physics), 104(24), 1-4. [245301].

https://doi.org/10.1103/PhysRevB.104.245301

This material is protected by copyright and other intellectual property rights, and duplication or sale of all or part of any of the repository collections is not permitted, except that material may be duplicated by you for your research use or educational purposes in electronic or print form. You must obtain permission for any other use. Electronic or print copies may not be offered, whether for sale or otherwise to anyone who is not an authorised user. 


\title{
Collective modes in an imbalanced nodal-line semimetal
}

\author{
SK Firoz Islam $\odot^{1}$ and Alexander A. Zyuzin ${ }^{1,2}$ \\ ${ }^{1}$ Department of Applied Physics, Aalto University, P. O. Box 15100, FI-00076 Aalto, Finland \\ ${ }^{2}$ Ioffe Physical-Technical Institute, 194021 St. Petersburg, Russia
}

(Received 15 October 2021; revised 23 November 2021; accepted 23 November 2021; published 3 December 2021)

\begin{abstract}
In this work, we investigate collective modes in a nodal line semimetal with two nodal lines that have opposite spin polarization in the presence of spin population imbalance. We find the components of the polarization operator, taking into account the electron-electron exchange interaction, and obtain the dispersion relations of collective modes for the bulk and surface states. There exist four modes in the bulk, among which one is gapless and other three are gapped. The gapless surface mode is sensitive to the boundary conditions.
\end{abstract}

DOI: 10.1103/PhysRevB.104.245301

Introduction. Recently, three-dimensional Dirac materials where conduction and valence bands merge along a line, so-called nodal line (or ring) semimetals (NLSMs), have attracted intense research interests due to their unique band structure [1-3]. The nodal line can be associated with the nontrivial Berry phase and the semimetal supports unusual surface states; for a recent review, see Ref. [4]. The existence of such band structure may be protected by time reversal, spatial inversion, or mirror symmetries [5-7]. A variety of materials have been reported [8-14]. The presence of an almost nondispersive Landau level was revealed in Ref. [15]. Angleresolved photoemission spectroscopy (ARPES) and quantum oscillation measurements have revealed the signatures of the nodal ring; see, for example, Refs. [9,16] and review [17].

The collective excitations are the fundamental optical properties of the fermionic systems. It was noted that the features of plasmon modes in gapless Dirac material like graphene [18] drastically differs from the electronic systems described by quadratic band structure. A similar investigation was also carried out in silicene [19], a two-dimensional puckered spin-orbit coupled honeycomb lattice of silicon atoms, exploring the spin- and valley-dependent plasmon modes. Recently, much attention has been paid to Dirac-Weyl materials in investigating plasmon modes, with a particular focus on the chiral anomaly [20-22]. Very recently, such collective modes have been studied in NLSMs as well [23,24]. It was shown that the plasmon modes in three-dimensional Weyl and NLSMs are gapped in the long-wavelength limit. The gap is determined by the respective density of states, which in the former case may be tuned by the parallel electric and magnetic field thanks to the chiral anomaly. However, the impact of electron-electron exchange interaction in the presence of population imbalance (either valley or spin, etc.) on collective excitations in Dirac materials is far from being understood.

The importance of the exchange interaction in the situation with nonequilibrium distribution of electron spin on spin-wave collective modes was first emphasized in Ref. [25]. Later, the spin waves in metals and semiconductors have been extensively investigated theoretically in Refs. [25-29].
Several experimental works were reported on the detection of spin waves in gaseous spin-polarized hydrogen, in the mixture of ${ }^{3} \mathrm{He}$ and ${ }^{4} \mathrm{He}$, polarized ${ }^{3} \mathrm{He}$ (see Ref. [29]). Recently, with analogy to spin, the exchange-interaction-induced valley wave in the Dirac materials with valley population imbalance has been discussed in Ref. [30]. Apart from graphene [31,32] and Weyl semimetals [33], the NLSM plasmon modes have been recently observed experimentally as reported in Ref. [34].

In this work, we consider a NLSM hosting two nodal rings with opposite spin orientation in the presence of the spin imbalance. The model of a semimetal with concentric loops that come from different spin channels in its band structure was introduced in Ref. [35]. We consider transverse spin waves mediated by the electron-electron exchange interaction. In this model, we find three gapped modes and a gapless mode. These modes are anisotropic and disperse quadratically in all direction in the long-wave limit. We also obtain a gapless spin-wave mode corresponding to the surface states.

Collective modes. We start with the effective Hamiltonian describing a model of semimetal with two nodal rings that have opposite spin polarization [35],

$$
H_{\eta}(\mathbf{k})=\epsilon\left(\frac{k_{\perp}^{2}}{Q^{2}}-1\right) \sigma^{x}+v_{z} k_{z} \sigma^{z}-\eta \frac{\lambda n}{2},
$$

where $\epsilon>0$ determines the top and bottom edges of the conductance and valence bands, respectively, and serves as an energy cutoff for the two-band model, $Q$ defines the radius of the nodal ring in the plane $k_{z}=0, v_{z}$ is the Fermi velocity along the $z$ direction, and $\sigma^{i}, i=(x, y, z)$ are the Pauli matrices acting on the orbital space. The last term describes the antisymmetric part of the electron-electron exchange interaction energy between two nodal rings, where the nodal ring spin-index is denoted by $\eta= \pm 1$. Note that the exchange interaction is taken to be momentum-independent within the simplest approximation for the Fourier component of screened Coulomb potential by $\lambda>0$. 
The low energy band dispersion is given by

$$
E_{b, \eta}=b \sqrt{\epsilon^{2}\left(k_{\perp}^{2} / Q^{2}-1\right)^{2}+v_{z}^{2} k_{z}^{2}}-\eta \frac{\lambda n}{2},
$$

where $b= \pm$ is the band index. The exchange-interactioninduced term splits the position of nodal rings in energy by $|\lambda n|$ with respect to each other. This is similar to the case of Dirac semimetals [30].

The exchange energy in Eq. (1) can be evaluated selfconsistently from the difference of particle densities between two nodal rings as

$$
n=\sum_{b= \pm} \int \frac{d^{3} k}{(2 \pi)^{3}}\left\{f\left[E_{b,+}\right]-f\left[E_{b,-}\right]\right\}
$$

where $f\left[E_{b, \eta}\right]=\left[1+e^{\left(E_{b, \eta}-\mu_{\eta}\right) / T}\right]^{-1}$ is the Fermi-Dirac distribution function in the nodal ring $\eta$ at temperature $T$. Summation is performed over two bands. The spin imbalance is described by the spin-dependent chemical potential as $\mu_{ \pm}=\mu \pm \delta \mu / 2$. As noted above, $\epsilon>\mu$ is the largest energy scale in the problem, so that the two-band approximation in formula (1) may be valid. For our study, we keep zero temperature $T=0$ throughout the discussion.

At $0<\mu_{ \pm} \pm \lambda n / 2<\epsilon$, the particle density imbalance is linearly proportional to $\delta \mu$ :

$$
n=\frac{\nu \delta \mu}{1-\lambda \nu}
$$

where $v=\mu Q^{2} / 4 \pi \epsilon v_{z}$ is the density of states at the Fermi level.

To proceed, let us consider a transverse magnetic field $\propto e^{i(\omega t-\mathbf{q} \cdot \mathbf{r})}$, which provides the electron transition between the two nodal rings with spin imbalance. The dispersion of collective modes is given by the poles of transverse susceptibility. The retarded and advanced Green's functions are given by $G_{\eta}^{R / A}(\mathbf{k}, \omega)=\left[\omega-H_{\eta}(\mathbf{k}) \pm i \delta\right]^{-1}$, i.e.

$G_{\eta}^{R / A}(\mathbf{k}, \omega)=\frac{1}{2} \sum_{b= \pm} \frac{\sigma_{0}+\frac{1}{E_{b, \eta}}\left[\epsilon\left(\frac{k_{\perp}^{2}}{Q^{2}}-1\right) \sigma^{x}+v_{z} k_{z} \sigma^{z}\right]}{\omega-E_{b, \eta} \pm i \delta}$,

and that can be used to evaluate the Keldysh Green's function as

$$
G_{\eta}^{K}(\mathbf{k}, \omega)=\left[1-2 f_{\eta}(\omega)\right]\left[G_{\eta}^{R}(\mathbf{k}, \omega)-G_{\eta}^{A}(\mathbf{k}, \omega)\right] .
$$

To obtain the dispersion relation of the inter-ring collective modes, we follow the standard random-phase approximation and seek the poles of the transverse susceptibility as $\operatorname{det}(1-$ $\lambda \Pi)=0$, where the inter-ring polarization operator is a $4 \times 4$ matrix, which is given by

$$
\begin{aligned}
\Pi_{a b, c d}= & \frac{i}{2} \int \frac{d^{3} k}{(2 \pi)^{3}} \frac{d \Omega}{2 \pi}\left[G_{-, a b}^{R}(k+q, \omega+\Omega) G_{+, c d}^{K}(k, \Omega)\right. \\
& \left.+G_{-, a b}^{K}(k+q, \omega+\Omega) G_{+, c d}^{A}(k, \Omega)\right] .
\end{aligned}
$$

It is convenient to write the polarization operator in new pseudospin representation $\Pi^{\alpha \beta}=\operatorname{Tr}_{2} \sigma_{b c}^{\alpha} \Pi_{a b, c d} \sigma_{d a}^{\beta} / 2$ where indices $\alpha, \beta$ take the values $(0, x, y, z)$, i.e., one singlet and three triplets, and $\operatorname{Tr}_{2}$ denotes trace of Pauli matrices.

The polarization operator in the new representation can be written through the sum of intraband and interband terms

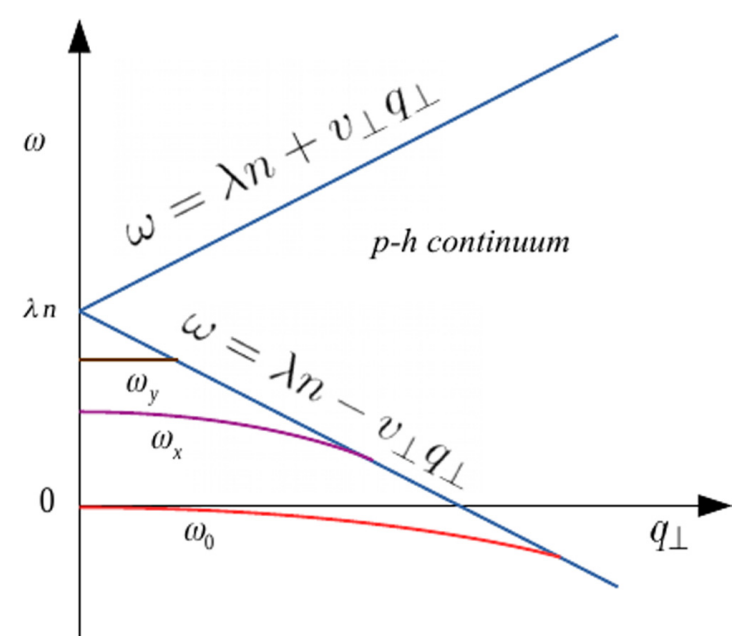

FIG. 1. Dispersions of the collective modes shown for the case $q_{z}=0$. The particle-hole continuum is depicted by the region confined by two lines $\omega=\lambda n \pm v_{\perp} q_{\perp}$.

as $\Pi^{\alpha \beta}=\Pi_{\text {intra }}^{\alpha \beta}+\Pi_{\text {inter }}^{\alpha \beta}$. The diagonal components which correspond to the interband contributions are obtained as $\Pi_{\text {inter }}^{00}=0, \Pi_{\text {inter }}^{x x}=2 \Pi_{\text {inter }}^{y y}=\Pi_{\text {inter }}^{z z}=v \ln (2 \epsilon / \mu) / 2$. On the other hand, the intraband components are obtained as

$$
\begin{aligned}
& \Pi_{i n t r a}^{00}=-v \frac{\delta \mu+\lambda n}{\omega-\lambda n}-v \frac{\delta \mu+\omega}{(\omega-\lambda n)^{3}}\left(v_{\perp}^{2} q_{\perp}^{2}+\frac{v_{z}^{2} q_{z}^{2}}{2}\right) \\
& \Pi_{i n t r a}^{x x}=-\frac{v}{2} \frac{\delta \mu+\lambda n}{\omega-\lambda n}-v \frac{\delta \mu+\omega}{(\omega-\lambda n)^{3}}\left(v_{\perp}^{2} q_{\perp}^{2}+\frac{v_{z}^{2} q_{z}^{2}}{2}\right)
\end{aligned}
$$

$\Pi_{\text {intra }}^{y y}=0$, and $\Pi_{\text {intra }}^{z z}=\Pi_{\text {intra }}^{x x}$, where $v_{\perp}=|\epsilon| / Q$. The off-diagonal intraband components are obtained up to linearin $q$ order as $\Pi_{\text {intra }}^{0 x}=\Pi_{\text {intra }}^{0 y}=0, \Pi_{\text {intra }}^{0 z}=\Pi_{\text {intra }}^{x z}=\Pi_{\text {intra }}^{y z}=$ $\Pi_{\text {intra }}^{z z}=\nu v_{z} q_{z} /(\omega-\lambda n)$, and $\Pi_{\text {intra }}^{x y}=0$. The off-diagonal components describe coupling between different modes.

Using the solution of the self-consistency equation (4) and assuming $\lambda n>v_{\perp} q_{\perp}, v_{z} q_{z}$ and $\lambda n>|\omega|$, the dispersion of collective modes can be obtained as

$$
\omega_{0}=-\frac{1-\lambda v}{\lambda n}\left(v_{\perp}^{2} q_{\perp}^{2}+\frac{1+\gamma}{2} v_{z}^{2} q_{z}^{2}\right),
$$

where $\quad \gamma=\lambda^{2} v^{2}[(1-\lambda v)(1-C)]^{-1} \ll 1 \quad$ with $\quad C=$ $2 \lambda \nu \ln (2 \epsilon / \mu)$. The other modes are given by

$$
\begin{aligned}
& \omega_{x}=\frac{\lambda n}{2}(1-C)-\frac{1-\lambda \nu}{\lambda n}\left(v_{\perp}^{2} q_{\perp}^{2}+\frac{1+\gamma}{2} v_{z}^{2} q_{z}^{2}\right), \\
& \omega_{y}=\lambda n\left(1-\frac{C}{2}\right)-2 \frac{(\lambda \nu)^{2}}{\lambda n} \frac{v_{z}^{2} q_{z}^{2}}{1-C},
\end{aligned}
$$

and $\omega_{z}=\omega_{x}$. We note that three modes $\left(\omega_{x}, \omega_{y}, \omega_{z}\right)$, corresponding to the triplet components of the polarization operator are gapped whereas the singlet component $\left(\omega_{0}\right)$ is gapless. The degree of gap is determined by the strength of electronelectron exchange interaction. The dispersions of collective modes are schematically shown in the Fig. 1. All these modes become damped while crossing the boundary of the particlehole continuum region, which, for example for $q_{z}=0$, is defined by $\lambda n-v_{\perp} q_{\perp} \leqslant \omega \leqslant \lambda n+v_{\perp} q_{\perp}$. 
Let us quickly comment on the zero-pumping case when $\delta \mu=0$. First, at $n=0$, the components of the polarization operator reduce to

$$
\Pi_{\text {intra }}^{00}=-\frac{v}{\omega^{2}}\left(v_{\perp}^{2} q_{\perp}^{2}+\frac{v_{z}^{2} q_{z}^{2}}{2}\right),
$$

and $\Pi_{\text {intra }}^{x x}=\Pi_{\text {intra }}^{y y}=\Pi_{\text {intra }}^{00}$. The collective mode with dispersion

$$
\omega=\sqrt{-\lambda \nu}\left(v_{\perp}^{2} q_{\perp}^{2}+\frac{v_{z}^{2} q_{z}^{2}}{2}\right)^{1 / 2}
$$

exists provided $\lambda<0$. Indeed, without the particle imbalance, the plasmon modes are expected, as shown in Ref. [24]. In this work, contrary to our study, the interaction term was taken to be $\lambda \sim 1 / q^{2}$, which leads to the gapped spectrum of the plasmon modes.

Surface states. One of the unique aspects of nodal-ring semimetals is the existence of the so-called drum-head surface states. Let us consider NLSM occupying a region $z>0$ with a boundary at $z=0$. We seek a wave function at the boundary as $\Psi=[A, B]^{T} e^{-z \alpha+i \mathbf{k}_{\perp} \cdot \mathbf{r}}$, where $\operatorname{Re} \alpha>0$ and $A, B$ are coefficients. The eigenvalue equation reads

$$
\left[\epsilon\left(k_{\perp}^{2} / Q^{2}-1\right) \sigma^{x}+i \alpha v_{z} \sigma^{z}\right] \Psi=\left(E_{\eta}+\eta \frac{\lambda_{s} n_{s}}{2}\right) \Psi .
$$

Here the exchange energy at the surface is denoted by $\lambda_{s}$ and $E_{\eta}$ is the energy eigenvalue for nodal ring $\eta= \pm 1$. Using the Hermiticity of the Hamiltonian one determines the boundary condition and obtains a wave function up to an arbitrary phase $\psi$ in the form

$$
\Psi \propto\left[1, e^{i \psi}\right]^{T} e^{-z \alpha+i \mathbf{k}_{\perp} \cdot \mathbf{r}} .
$$

Generally $\psi$ may be a function of position at the boundary. Here we consider it to be coordinate-independent. Separating the real and imaginary parts from the equation $\left[i v_{z} \alpha-\left(E_{\eta}+\right.\right.$ $\left.\left.\eta \lambda_{s} n / 2\right)\right]+\epsilon\left(k_{\perp}^{2} / Q^{2}-1\right) e^{i \psi}=0$, one gets the dispersion of surface state in the form

$$
E_{\eta}=\epsilon\left(k_{\perp}^{2} / Q^{2}-1\right) \cos \psi-\eta \frac{\lambda_{s} n_{s}}{2},
$$

provided the condition

$$
\alpha v_{z} \equiv-\epsilon\left(k_{\perp}^{2} / Q^{2}-1\right) \sin \psi>0
$$

holds. At $\psi= \pm \pi / 2$, the flat band surface state is strictly localized at the surface, whereas the state is delocalized for $\psi=0$. Without loss of generality, we consider $\psi \in[0, \pi / 2)$.

Let us now discuss the collective modes for surface states in the presence of population imbalance. We neglect the interference between bulk and localized surface states because we are looking at two limiting cases only. Following the same approach as in the bulk we obtain the density imbalance at the surface. The chemical potential for the surface states with spin imbalance is taken to be $\mu_{\eta}=\mu+\eta \delta \mu / 2$. One obtains $n_{s}=v_{s} \delta \mu /\left(1-\lambda_{s} v_{s}\right)$ provided $\mu<0$ and $|\mu|<$ $\epsilon \cos \psi$. Here $v_{s}=Q^{2} /(4 \pi \epsilon \cos \psi)$ is the two-dimensional (2D) surface density of states. The polarization operator is obtained as

$$
\begin{aligned}
\Pi_{s}= & -v_{s} \frac{\delta \mu+\lambda_{s} n_{s}}{\omega-\lambda_{s} n_{s}} \\
& -v_{s}\left(1-\frac{|\mu|}{\epsilon \cos \psi}\right) \frac{\omega+\delta \mu}{\left(\omega-\lambda_{s} n_{s}\right)^{3}}\left(v_{\perp} q_{\perp} \cos \psi\right)^{2} .
\end{aligned}
$$

Hence, the surface mode dispersion at $\lambda_{s} v_{s}>v_{\perp} q_{\perp}$ and $\lambda_{s} v_{s}>|\omega|$ is given by

$$
\omega=-\left(1-\frac{|\mu|}{\epsilon \cos \psi}\right)\left(1-\lambda_{s} v_{s}\right) \cos ^{2}(\psi) \frac{v_{\perp}^{2} q_{\perp}^{2}}{\lambda_{s} n_{s}},
$$

which is quadratic in wave vector and the slope is strongly determined by the properties of the surface. Note that, similar to $\omega_{0}$ in Eq. (9), the surface mode varies inversely with $\lambda_{s} n_{s}$. However, the mode strongly depends on the properties of the boundary, vanishing in the limit $\cos \psi \rightarrow|\mu| / \epsilon$. Now we comment on the dispersion in the absence of imbalance, i.e., $\delta \mu=0$. In this case, the polarization operator reduces to

$$
\Pi_{s}=-v_{s}\left(1-\frac{|\mu|}{\epsilon \cos \psi}\right)\left(\frac{v_{\perp} q_{\perp} \cos \psi}{\omega}\right)^{2},
$$

which leads the spectrum as

$$
\omega=\sqrt{-\lambda_{s} v_{s}}\left(1-\frac{|\mu|}{\epsilon \cos \psi}\right)^{1 / 2} v_{\perp} q_{\perp} \cos \psi,
$$

indicating that surface mode exists only for $\lambda_{s}<0$. It is also interesting to note that the mode is linearly dispersive, whereas in the presence of imbalance, it disperses quadratically.

Effects of tilt. Now we comment on the possible effects of a time-reversal symmetry-breaking perturbation on the spectrum of the mode. The Hamiltonian can be written for a tilted nodal ring as

$$
H_{\eta}=\epsilon\left(\frac{k_{\perp}^{2}}{Q^{2}}-1\right)\left(\sigma^{x}+\eta t_{\perp}\right)+v_{z} k_{z}\left(\sigma^{z}+\eta t_{z}\right)-\eta \frac{\lambda n}{2} .
$$

The spin index $\eta$ indicates that the two nodal rings are tilted in opposite directions. We note that, for the weak tilting $\left|t_{\perp}\right| \ll$ $1,\left|t_{z}\right| \ll 1$, the intraband component of the polarization operator is slightly affected in terms of the coefficients at $q_{\perp}, q_{z}$. Hence, the tilting modifies velocities $v_{\perp}$ and $v_{z}$ of valley-wave modes but not the wave-vector power-law dependence. It is also noteworthy to mention that the weak tilting does not affect the mode gap.

Finally, we draw a comparison with the valley wave in gapless Dirac-Weyl systems, studied recently in Ref. [30]. We note that the gapless collective mode in the presence of the population imbalance in NLSMs, graphene, or Weyl semimetals exhibit almost similar behavior except the degree of curvature, which depends on system parameters. However, the degree of gap in the spectrum is strongly determined by the dimension and exchange interaction. For example, we note that the gapless mode exists in the NLSM case for any sign of the relative Berry flux of the two nodal rings. This is not the case for three-dimensional Dirac-Weyl materials, as discussed in Ref. [30]. Another important difference with Dirac-Weyl systems is the feature of collective modes which arise due to surface states. 
Although collective charge dynamics of Fermi arc surface states in Weyl semimetals has been studied [36,37], to the best of our knowledge, the exchange interaction mediated collective modes in Fermi arcs surface states have not been discussed yet. To support the population-imbalance scenario and resulting collective modes, the system has to possess at least two orthogonal surface states. This criteria may be satisfied, for example, in antiferromagnetic Dirac semimetals, where counterpropagating Fermi arc surface states are expected [38].

Summary. To conclude, we investigate the electronelectron exchange-interaction-generated collective modes in nodal-line semimetals which arise due to the population im- balance between two nodal rings. We evaluate the transverse polarization function, which enables us to obtain the dispersion relations of internode collective modes. We find a gapless mode with a quadratic dispersion relation for both the bulk and surface states. Very recently, plasmon modes have been observed in NLSMs [34] in an equilibrium situation, which can be extended further to spin-nonequilibrium cases with double or multiple nodal rings to realize our findings. We also reveal that the plasmon dispersion for surface states switches to linearly dispersive from quadratically dispersive while turning off the imbalance between two rings.

Acknowledgment. This work is supported by the Academy of Finland Grant No. 308339.
[1] G. P. Mikitik and Y. V. Sharlai, Phys. Rev. Lett. 82, 2147 (1999).

[2] T. Heikkilä and G. E. Volovik, JETP Lett. 93, 59 (2011).

[3] A. A. Burkov, M. D. Hook, and L. Balents, Phys. Rev. B 84, 235126 (2011).

[4] S.-Y. Yang, H. Yang, E. Derunova, S. S. P. Parkin, B. Yan, and M. N. Ali, Adv. Phys.: X 3, 1414631 (2018).

[5] Y. Kim, B. J. Wieder, C. L. Kane, and A. M. Rappe, Phys. Rev. Lett. 115, 036806 (2015).

[6] C. Fang, Y. Chen, H.-Y. Kee, and L. Fu, Phys. Rev. B 92, 081201(R) (2015).

[7] Y.-H. Chan, C.-K. Chiu, M. Y. Chou, and A. P. Schnyder, Phys. Rev. B 93, 205132 (2016).

[8] G. Bian, T.-R. Chang, H. Zheng, S. Velury, S.-Y. Xu, T. Neupert, C.-K. Chiu, S.-M. Huang, D. S. Sanchez, I. Belopolski, N. Alidoust, P.-J. Chen, G. Chang, A. Bansil, H.-T. Jeng, H. Lin, and M. Z. Hasan, Phys. Rev. B 93, 121113(R) (2016).

[9] J. Hu, Z. Tang, J. Liu, X. Liu, Y. Zhu, D. Graf, K. Myhro, S. Tran, C. N. Lau, J. Wei, and Z. Mao, Phys. Rev. Lett. 117, 016602 (2016).

[10] D. Takane, Z. Wang, S. Souma, K. Nakayama, C. X. Trang, T. Sato, T. Takahashi, and Y. Ando, Phys. Rev. B 94, 121108(R) (2016).

[11] X.-B. Wang, X.-M. Ma, E. Emmanouilidou, B. Shen, C.-H. Hsu, C.-S. Zhou, Y. Zuo, R.-R. Song, S.-Y. Xu, G. Wang, L. Huang, N. Ni, and C. Liu, Phys. Rev. B 96, 161112(R) (2017).

[12] Z. Liu, R. Lou, P. Guo, Q. Wang, S. Sun, C. Li, S. Thirupathaiah, A. Fedorov, D. Shen, K. Liu, H. Lei, and S. Wang, Phys. Rev. X 8, 031044 (2018).

[13] D. Takane, K. Nakayama, S. Souma, T. Wada, Y. Okamoto, K. Takenaka, Y. Yamakawa, A. Yamakage, T. Mitsuhashi, K. Horiba et al., npj Quantum Mater. 3, 1 (2018).

[14] J. Behrends, J.-W. Rhim, S. Liu, A. G. Grushin, and J. H. Bardarson, Phys. Rev. B 96, 245101 (2017).

[15] J.-W. Rhim and Y. B. Kim, Phys. Rev. B 92, 045126 (2015).

[16] N. Kumar, K. Manna, Y. Qi, S.-C. Wu, L. Wang, B. Yan, C. Felser, and C. Shekhar, Phys. Rev. B 95, 121109(R) (2017).

[17] B. Q. Lv, T. Qian, and H. Ding, Rev. Mod. Phys. 93, 025002 (2021).

[18] E. H. Hwang and S. Das Sarma, Phys. Rev. B 75, 205418 (2007).
[19] B. Van Duppen, P. Vasilopoulos, and F. M. Peeters, Phys. Rev. B 90, 035142 (2014).

[20] S. Das Sarma and E. H. Hwang, Phys. Rev. Lett. 102, 206412 (2009).

[21] J. Zhou, H.-R. Chang, and D. Xiao, Phys. Rev. B 91, 035114 (2015).

[22] I. Panfilov, A. A. Burkov, and D. A. Pesin, Phys. Rev. B 89, 245103 (2014).

[23] J.-W. Rhim and Y. B. Kim, New J. Phys. 18, 043010 (2016).

[24] Z. Yan, P.-W. Huang, and Z. Wang, Phys. Rev. B 93, 085138 (2016).

[25] A. Aronov, Zh. Eksp. Teor. Fiz. 73, 577 (1977) [Sov. Phys. JETP 46, 301 (1977)].

[26] C. Deutsch, F. Ramirez-Martinez, C. Lacroûte, F. Reinhard, T. Schneider, J.-N. Fuchs, F. Piéchon, F. Laloë, J. Reichel, and P. Rosenbusch, Phys. Rev. Lett. 105, 020401 (2010).

[27] K. S. Bedell and H. P. Dahal, Phys. Rev. Lett. 97, 047204 (2006).

[28] A. A. Zyuzin and A. Y. Zyuzin, Europhys. Lett. 90, 67007 (2010).

[29] E. Bashkin, Sov. Phys. Usp. 29, 238 (1986).

[30] A. A. Zyuzin and A. Y. Zyuzin, Phys. Rev. B 100, 121402(R) (2019)

[31] L. Ju, B. Geng, J. Horng, C. Girit, M. Martin, Z. Hao, H. A. Bechtel, X. Liang, A. Zettl, Y. R. Shen et al., Nat. Nanotechnol. 6, 630 (2011).

[32] P. Di Pietro, M. Ortolani, O. Limaj, A. Di Gaspare, V. Giliberti, F. Giorgianni, M. Brahlek, N. Bansal, N. Koirala, S. Oh et al., Nat. Nanotechnol. 8, 556 (2013).

[33] G. Chiarello, J. Hofmann, Z. Li, V. Fabio, L. Guo, X. Chen, S. Das Sarma, and A. Politano, Phys. Rev. B 99, 121401(R) (2019).

[34] S. Xue, M. Wang, Y. Li, S. Zhang, X. Jia, J. Zhou, Y. Shi, X. Zhu, Y. Yao, and J. Guo, Phys. Rev. Lett. 127, 186802 (2021).

[35] R.-W. Zhang, Z. Zhang, C.-C. Liu, and Y. Yao, Phys. Rev. Lett. 124, 016402 (2020).

[36] E. V. Gorbar, V. A. Miransky, I. A. Shovkovy, and P. O. Sukhachov, Phys. Rev. B 99, 155120 (2019).

[37] F. Adinehvand, Z. Faraei, T. Farajollahpour, and S. A. Jafari, Phys. Rev. B 100, 195408 (2019).

[38] P. Tang, Q. Zhou, G. Xu, and S.-C. Zhang, Nat. Phys. 12, 1100 (2016). 